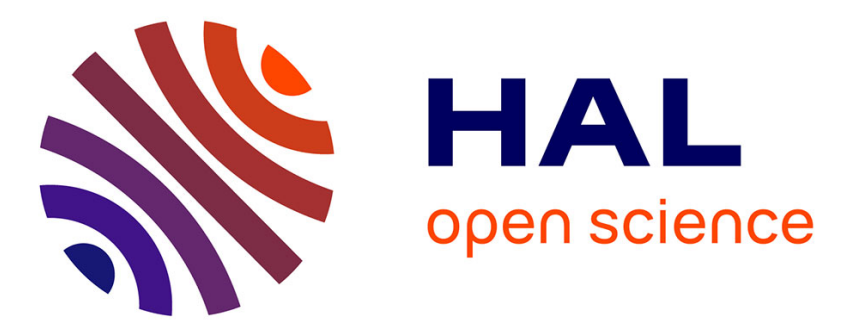

\title{
Mieux prévoir les crues nivales : évaluation de prévisions probabilistes de débit sur des bassins versants de montagne français
}

Pierre Nicolle, Audrey Valéry, M.H. Ramos, Charles Perrin, Vazken Andréassian

\section{To cite this version:}

Pierre Nicolle, Audrey Valéry, M.H. Ramos, Charles Perrin, Vazken Andréassian. Mieux prévoir les crues nivales: évaluation de prévisions probabilistes de débit sur des bassins versants de montagne français. La Houille Blanche - Revue internationale de l'eau, 2012, 2, pp.26-33. 10.1051/lhb/2012012 . hal-02597314

\section{HAL Id: hal-02597314 \\ https://hal.inrae.fr/hal-02597314}

Submitted on 15 May 2020

HAL is a multi-disciplinary open access archive for the deposit and dissemination of scientific research documents, whether they are published or not. The documents may come from teaching and research institutions in France or abroad, or from public or private research centers.
L'archive ouverte pluridisciplinaire HAL, est destinée au dépôt et à la diffusion de documents scientifiques de niveau recherche, publiés ou non, émanant des établissements d'enseignement et de recherche français ou étrangers, des laboratoires publics ou privés. 


\title{
Mieux prévoir les crues nivales : évaluation de prévisions probabilistes de débit sur des bassins versants de montagne français
}

\author{
Pierre NICOLLE ${ }^{1}$, Audrey VALERY ${ }^{1,2}$, Maria-Helena RAMOS ${ }^{1}$, Charles PERRIN ${ }^{1}$, Vazken ANDREASSIAN ${ }^{1}$ \\ ${ }^{1}$ Cemagref / Irstea, Antony, UR HBAN - e-mail : pierre.nicolle@irstea.fr \\ 2.Coyne et Bellier - e-mail : audrey.valery@gdfsuez.com
}

\begin{abstract}
RÉSUMÉ. - La prévision hydrologique présente des enjeux considérables concernant la sécurité des personnes et des biens, ainsi que la gestion des ressources en eau. Bien prévoir les débits est essentiel pour prendre les bonnes décisions. Certains types de bassins versants présentent cependant des défis particuliers : c'est notamment le cas des espaces montagneux, où l'importante présence de neige et les difficultés de mise en place de réseaux de mesures compliquent la simulation et la prévision opérationnelle des débits. Cette étude vise à évaluer les prévisions hydrologiques obtenues à partir d'une approche de modélisation qui intègre un module neige au sein d'un modèle de prévision hydrologique. Le module CemaNeige [Valéry, 2010] et le modèle de prévision hydrologique GR3P à trois paramètres [Tangara, 2005] ont été utilisés. L'évaluation des prévisions du modèle intégré GR5P à cinq paramètres (GR3P avec module neige) a été réalisée au pas de temps journalier et sur un échantillon de 176 bassins versants français situés dans des massifs montagneux. Le modèle hydrologique utilise en entrée quatre années de prévisions du système de prévision d'ensemble PEARP de Météo-France (2005-2009). Onze scénarios équiprobables de débits sont prévus à chaque jour et pour deux échéances de prévision $(\mathrm{j}+1$ et $\mathrm{j}+2)$. Les débits prévus sont évalués par rapport aux débits observés de la banque HYDRO. Les résultats illustrent l'importance du traitement du stockage et de la fonte de la neige, notamment pour les bassins versants à régime dominant de type nival et de transition. L'amélioration des débits prévus en tenant compte de la neige est encore plus importante lorsque l'échéance de prévision augmente.
\end{abstract}

Mots clefs : modélisation hydrologique, module neige, prévision d'ensemble

\section{Better predicting snowmelt-induced floods : evaluation of probabilistic streamflow forecasts on mountainous catchments in France}

\begin{abstract}
Hydrological forecasting presents considerable challenges concerning the protection of people and properties, as well as the management of water resources. Good streamflow estimates are essential to make the right decisions. However, some catchments present particular challenges : this is the case of mountainous areas, where the occurrence of snow and the difficulties of implementing observational networks complicate flow modelling and operational flow forecasting. This study aims to evaluate flow forecasts obtained from a modelling system that integrates a snow module into a hydrological forecasting model. The CemaNeige snow routine [Valéry, 2010] and the three-parameter GR3P hydrological model [Tangara, 2005] were used. The analysis of the streamflow forecasts from the five-parameter integrated system GR5P (GR3P with snow modelling) was performed at the daily time step and on a set of 176 French catchments located in mountainous areas. The hydrological model was fed with four years of PEARP ensemble forecasts from Météo-France (2005-2009). Eleven equally probable scenarios of streamflow are predicted daily and for two forecasting lead times $(\mathrm{d}+1$ and $\mathrm{d}+2)$. Forecast discharges are evaluated against observed discharges from the HYDRO database. The results illustrate the importance of the treatment of snow storage and melting, especially for nival and transitional hydrological regimes. The improvement by accounting for snow becomes even more significant when the forecasting lead time increases.
\end{abstract}

Key words : hydrological modelling, snow routine, ensemble forecasting

\section{INTRODUCTION}

La prise en compte de la neige dans le fonctionnement hydrologique des bassins versant de montagne est une étape essentielle à la modélisation des débits. Depuis longtemps, les hydrologues se sont attachés à prendre en compte ce facteur et des outils de modélisation du stockage et de la fonte de la neige ont été développés et incorporés à la modélisation pluie-débit. De manière générale, ces outils cherchent à bien représenter l'accumulation des précipitations neigeuses sur le bassin, l'évolution spatio-temporelle de la couverture neigeuse et la remobilisation de l'eau dans le bassin lors de la fonte. Ils sont utilisés conjointement au modèle hydrologique pour fournir une modélisation complète du bassin versant.

Il est possible de dégager deux grandes étapes à la modélisation hydro-nivale (voir la synthèse proposée par Klemeŝ [1990] et les nombreuses références dans Gottardi [2009] et Valéry [2010]). Une première étape consiste à quantifier et spatialiser les données d'entrée en altitude pour améliorer 
leur représentation au sein des modèles hydrologiques. De nombreux travaux ont été effectués sur les températures et les précipitations, notamment à l'aide de régressions linéaires pour caler un gradient altitudinal, de méthodes d'interpolation par krigeage, ou des méthodes d'estimation par bilan hydrique.

Une fois les données de précipitation et température spatialisées, la seconde étape concerne la modélisation de la neige au sol : le calcul de la fonte et de l'évolution spatiotemporelle de la couverture neigeuse à l'échelle du bassin. Deux grandes approches pour la production de la lame de fonte issue du manteau neigeux ressortent (voir une présentation détaillée dans Valéry [2010]) : les approches physiques par bilan énergétique (e.g. [Garen and Marks, 2005] et bilan de masse (e.g. [Lehning et al., 2006]), qui permettent de détailler les processus physiques liés à la fonte, et les approches conceptuelles par degrés-jour (e.g. [Zappa et al., 2003] en association avec le modèle hydrologique distribué PREVAH), qui s'appuient sur la température de l'air et des paramètres tels le facteur de fonte $\left(\mathrm{mm} .{ }^{\circ} \mathrm{C}^{-1}\right)$ et la température seuil au-dessus de laquelle une lame de fonte est calculée. Pour ce qui est de l'évolution spatiale du couvert neigeux, nous retiendrons les approches par modélisation du manteau neigeux selon des zones ou bandes d'altitude (discrétisation du bassin versant modélisé) et les stratégies basées sur l'établissement de courbes d'épuisement du stock de neige à partir d'observations de surface (données obtenues souvent à l'aide d'outils de télédétection). Pour le routage de la lame de fonte jusqu'à l'exutoire du bassin, les approches de la littérature adoptent souvent une solution simplifiée qui consiste à ajouter la lame de fonte aux précipitations liquides du bassin et à soumettre le total ainsi obtenu à la fonction de routage spécifique du modèle pluie-débit utilisé. Enfin, on notera que les approches de modélisation peuvent également être utilisées de façon combinée et que le choix d'une stratégie de modélisation dépendra de plusieurs facteurs tels la disponibilité des données et les contraintes de mise en œuvre en contexte opérationnel.

Ainsi, de nombreux modules de prise en compte de la neige ont été développés et intégrés à des modèles hydrologiques pour des objectifs divers (e.g. ingénierie et dimensionnement d'ouvrages, estimation de la ressource en eau, simulation et prévision des débits). Les travaux de Valéry [2010] ont comparé sept modules neige existants dans la littérature, testés en association à quatre modèles hydrologiques, sur 380 bassins versants. Il en ressort que même des structures simples peuvent apporter un gain significatif de performance à la simulation des débits dans des bassins de montagne. Les modules neiges testés étaient ceux du modèle MORDOR (selon une approche globale de la neige [Garçon, 1996, 1999] ou distribuée [Paquet, 2004]), le module NAM de la plateforme MIKE [DHI, 2009], le module CEQUEAU [Morin, 2002], le module MOHYSE [Turcotte et al., 2007] et le module neige du modèle hydrologique HBV (Bergström [1975], Lindström et al. [1997]).

Dans le but de mettre en place un outil de modélisation parcimonieux et robuste pour les besoins opérationnels, un module neige simple et général, nécessitant peu de données d'entrée pour son fonctionnement, baptisé CemaNeige, a été récemment proposé par Valéry [2010]. Basé sur la spatialisation des températures et des précipitations, et sur une approche par degrés-jour, ce module, à deux paramètres libres, a été intégré à des modèles hydrologiques globaux et testé sur un large échantillon de bassins versants. Les résultats obtenus ont montré une bonne performance en simulation hydrologique. Le but de la présente étude est d'évaluer les performances de ce module neige en prévision hydrologique. Pour cela, dans un premier temps, le module neige a été intégré au modèle de prévision pluie-débit GR3P [Tangara, 2005], utilisé par plusieurs Services de Prévision des Crues (SPC) en France [Nicolle, 2010]. Ensuite, les prévisions émises sur un grand nombre de bassins versants ont été évaluées à l'aide de différents critères d'évaluation de la qualité des prévisions. L'évaluation a été conduite, d'une part, en utilisant les précipitations observées en entrée et, d'autre part, en utilisant une archive de prévisions météorologiques de Météo-France. L'objectif in fine est de tester la modélisation intégrée pour son utilisation en contexte opérationnel, alors même que les services opérationnels disposent souvent d'outils et données limités pour améliorer les performances de leurs prévisions de débits dans les bassins de montagne.

\section{DONNEES ET METHODE}

\section{II.1. Données}

\section{II.1.1. Données observées}

Les précipitations et les températures de l'air proviennent de la base de données issue du système d'analyse météorologique SAFRAN de Météo-France (Quintana-Segui et al. [2008], Vidal et al. [2009]). L'évapotranspiration, utilisée en entrée du modèle hydrologique, est celle calculée à partir de la formule d'Oudin et al. [2005]. Les débits journaliers sont issus de la banque de données HYDRO (www.hydro. eaufrance.fr).

\section{II.1.2. Prévisions météorologiques}

Les prévisions de précipitations (pluie et neige) et de températures de l'air de la PEARP (Prévision d'Ensemble ARPège) de Météo-France [Nicolau, 2002] sont utilisées. Des études antérieures basées sur les prévisions PEARP ont montré des bonnes performances du système pour la prévision de débits à courte échéance, même si le système reste perfectible, notamment en termes d'amélioration de la dispersion des scenarios prévus (Thirel et al., [2008], Randrianasolo et al. [2010]). Ici, les valeurs des mailles de la PEARP $\left(8^{\prime} 8 \mathrm{~km}^{2}\right)$ ont été agrégées sur les bassins versants de l'étude (moyenne pondérée par les superficies des mailles appartenant au bassin). Les prévisions, lancées à $18 \mathrm{~h}$ le jour j d'émission de la prévision et disponibles au pas de temps tri-horaire, ont été agrégées pour fournir des prévisions journalières pour l'échéance $1(\mathrm{j}+1)$ et l'échéance $2(\mathrm{j}+2)$. Les onze scénarios de la PEARP ont été utilisés en entrée du modèle hydrologique GR3P et les onze prévisions hydrologiques d'ensemble résultantes ont été évaluées.

\section{II.1.3. Bassins versants d'étude}

Les 176 bassins versants étudiés sont des bassins de montagne français, localisés sur les reliefs du Massif Central, du Jura, des Alpes et des Pyrénées (figure 1). Ils présentent les avantages suivants pour notre étude :

- des bassins peu à fortement influencés par la neige ;

- une limitation de phénomènes qui pourraient «bruiter» le signal de débit lié à la neige : pourcentage de surface glaciaire nul ou limité ; superficie des surfaces en eau (lacs) limitée ; caractère « naturel » des bassins, c'est-à-dire sans dérivation ou exploitation anthropique significative, privilégié ;

- une certaine hétérogénéité de l'échantillon en termes de caractéristiques physiographiques, climatiques et hydrologiques, comme le montre le tableau 1. 


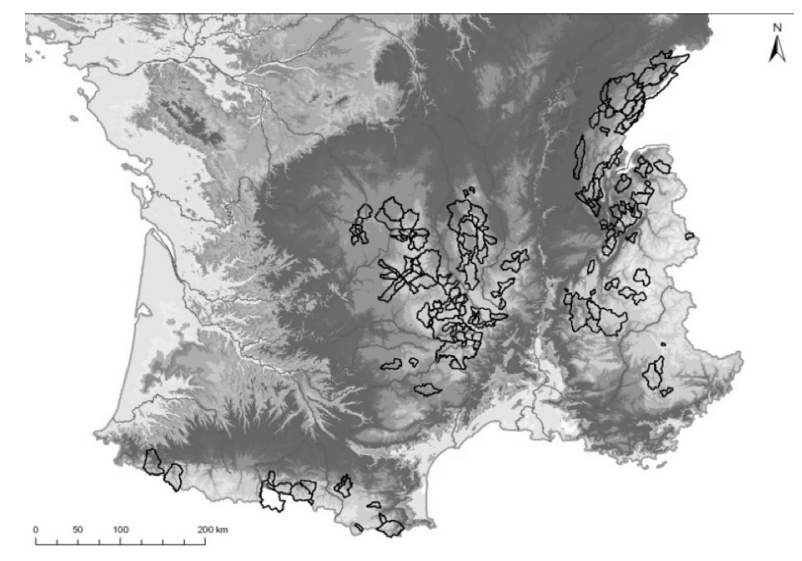

Figure 1 : Carte de localisation des 176 bassins versants étudiés

Afin de caractériser davantage notre échantillon de bassins versants, nous avons choisi de les différencier en fonction de leur régime hydrologique. L'objectif est de pouvoir évaluer les performances du modèle de prévision selon différents degrés d'influence du stockage et de la fonte de la neige sur les régimes hydrologiques. Compte tenu de l'importance de notre échantillon et de la complexité de caractériser les facteurs à l'origine des régimes hydrologiques, nous avons cherché à nous baser sur une classification objective établie par Sauquet [2006]. Le manque de représentativité de certains régimes hydrologiques dans notre échantillon nous a amené à effectuer un regroupement en trois types (figure 2) : les bassins à régime pluvial représentent $73.8 \%$ de l'échantillon (130 bassins), les régimes de transition sont représentés par 38 bassins versants et les bassins à régime nival représentent $4.6 \%$ de l'échantillon ( 8 bassins).

\section{II.2. Modèles utilisés}

\section{II.2.1. Le modèle de prévision hydrologique : GR3P}

Nous utilisons le modèle de prévision hydrologique GR3P au pas de temps journalier, initialement proposé par Tangara [2005] et adapté pour la prévision d'ensemble [Ramos et al., 2008]. C'est un modèle global à trois paramètres, qui possède une structure à réservoir et fonctionne en continu. Dans le modèle GR3P, la pluie est d'abord neutralisée par l'évapotranspiration potentielle. Le réservoir de production gère ensuite la partition des précipitations entre alimentation de l'écoulement (pluie nette) et alimentation du réservoir, ce qui permet d'assurer un suivi (via l'état de ce réservoir) des conditions d'humidité du bassin. Cette pluie nette est ajustée pour permettre notamment de tenir compte d'éventuels échanges avec des nappes profondes et/ou des bassins voisins. Le transfert de l'eau est ensuite assuré par un hydrogramme unitaire qui permet de prendre en compte le décalage temporel entre la pluie et le débit. Cet hydrogramme est associé à un réservoir de routage quadratique pour fournir le débit de sortie à l'exutoire du bassin. L'assimilation du dernier débit observé est réalisée par mise à jour directe du contenu de ce réservoir. Le niveau du réservoir est ainsi recalculé pour générer un débit égal au dernier débit observé à l'instant de la prévision.

\section{II.2.2. Le module de prise en compte de la neige: CemaNeige}

CemaNeige est un module de prise en compte de la neige développé par Valéry [2010]. Il prend en compte une discrétisation spatiale du bassin versant en cinq zones d'altitude d'égale surface. Pour chacune des zones d'altitude, la température et la précipitation du bassin sont extrapolées à l'aide de corrections altitudinales optimisées. La nature des précipitations (solide ou liquide) se fait suivant l'altitude médiane $\mathrm{du}$ bassin et les températures (minimale et maximale) du pas de temps et de la zone considérés. Les précipitations solides sont intégrées à un réservoir qui représente le manteau neigeux. La lame de fonte potentielle est estimée selon la méthode degrés-jour, à partir de l'état thermique du manteau neigeux et de la température. La lame de fonte réelle est ensuite calculée en fonction de la fonte potentielle, de la surface enneigée et, lorsque le stock de neige est faible, de la vitesse minimale de fonte. Les lames de fonte de chaque bande d'altitude sont agrégées et ajoutées aux précipitations liquides en entrée du modèle hydrologique.

Le module neige possède ainsi deux variables descriptives internes pour chacune des zones d'altitude (le stock de neige et l'état thermique du stock) et deux paramètres libres identiques pour les cinq zones à caler : le facteur de fonte et le coefficient de pondération de l'état thermique du manteau. De plus, quatre paramètres fixes ont été déterminés empiriquement. Ils concernent l'intervalle de température dans lequel les fractions liquide et solide des précipitations sont estimées (si l'estimation est faite à partir de températures journalières moyennes), la température de fonte (fixée à $0{ }^{\circ} \mathrm{C}$ ), le stock de neige à partir duquel toute la zone d'altitude considérée est recouverte de neige, et la vitesse de fonte minimale (pour plus de détail, voir Valéry [2010]).

Dans cette étude, pour chaque bassin versant, les cinq paramètres libres du modèle intégré GR5P (c'est-à-dire, les trois paramètres du modèle GR3P et les deux paramètres du module neige) ont été calés simultanément. L'optimisation a été faite en minimisant les erreurs quadratiques moyennes (RMSE) entre les débits observés et simulés, sur les données disponibles entre août 1958 et juillet 2000. Les paramètres ne sont pas recalibrés lorsque le forçage météorologique est issu des prévisions météorologiques d'ensemble.

\section{II.3. Méthode d'évaluation}

L'évaluation a été menée en deux étapes. Dans un premier temps, les performances du modèle ont été évaluées en prenant comme scénario de précipitation future les précipitations observées a posteriori. Cette évaluation

Tableau 1: Principales caractéristiques des bassins versants étudiés (entre crochets, valeurs minimales et maximales et, en bas, valeur médiane sur 176 bassins).

\begin{tabular}{|c|c|c|c|c|c|c|c|}
\hline $\begin{array}{c}\text { Superficie } \\
\left(\mathbf{k m}^{2}\right)\end{array}$ & $\begin{array}{c}\mathbf{Z m e d} \\
(\mathbf{m})\end{array}$ & $\begin{array}{c}\Delta \mathbf{Z} \\
(\mathbf{m})\end{array}$ & $\begin{array}{c}\mathbf{P} \\
(\mathbf{m m} / \mathbf{a n})\end{array}$ & $\begin{array}{c}\mathbf{Q} \\
(\mathbf{m m} / \mathbf{a n})\end{array}$ & $\begin{array}{c}\mathbf{E T P} \\
(\mathbf{m m} / \mathbf{a n})\end{array}$ & $\begin{array}{c}\text { Neige } \\
(\mathbf{m m} / \mathbf{a n})\end{array}$ & \% Neige \\
\hline$[5 ; 2210]$ & {$[386 ; 2681]$} & {$[133 ; 2644]$} & {$[763 ; 2287]$} & {$[233 ; 2070]$} & {$[280 ; 714]$} & {$[12 ; 740]$} & {$[1 ; 51]$} \\
105 & 1035 & 833 & 1357 & 671 & 588 & 87 & 7 \\
\hline
\end{tabular}


en contrôle, avec le modèle forcé par les observations, a été faite à l'aide du critère de persistance borné C2MP (Tangara [2005], Mathevet et al. [2006]), qui compare le modèle de prévision à un modèle naïf qui prévoit toujours le dernier débit observé. Ce critère est défini sur ]-1, +1] ; la valeur 1 indique un accord parfait entre valeurs observées et prévues.

Dans un deuxième temps, les performances du modèle ont été évaluées en mode prévision. Le modèle est forcé par les scénarios de prévision météorologique d'ensemble du système PEARP. Ainsi, pour chaque jour de prévision, on dispose d'un ensemble de 11 scénarios de débits prévus à deux échéances journalières $(j+1$ et $\mathrm{j}+2)$. Puisqu'il s'agit de l'évaluation de plusieurs scénarios de prévision, nous utilisons des critères statistiques de performance souvent appliqués aux prévisions probabilistes. Ici, nous présentons les résultats obtenus avec le critère RPS (Score de probabilité ordonnée) et avec deux critères qui visent à qualifier les performances d'un système de prévision par rapport à un évènement particulier, défini par le dépassement (ou nondépassement) d'un seuil critique : le Brier Score (BS) et le score CSI (Critical Success Index). Dans le but d'évaluer la qualité des prévisions de crues, nous présentons les résultats obtenus en considérant le dépassement du seuil critique donné par le quantile $90 \%$ de la distribution des débits observés, calculé sur la période d'évaluation. Les scores utilisés sont brièvement présentés ci-après. Pour plus de détails, on se reportera à Wilks [2011] et Nicolle [2010].

Le principe du score de probabilité ordonnée (RPS) consiste à comparer les probabilités au non-dépassement prévues et observées sur un ensemble de catégories (ou classes de débits). Ici, les $\mathrm{K}+1=10$ catégories sont les intervalles définis par les K quantiles Q10, Q20...Q90 \% de la distribution des débits observés, calculés sur la période d'évaluation. Le score RPS est en quelque sorte une mesure de " distance » entre la distribution de probabilité cumulative des prévisions et la valeur observée. Un score RPS parfait vaut zéro.

Le Brier Score (BS) est un critère similaire à l'erreur quadratique moyenne, mais dans l'espace des probabilités : il compare les probabilités prévues et observées. Il est estimé en évaluant la somme des différences au carré entre les probabilités de prévision (pourcentage de scénarios de débits prévus qui dépassent le seuil critique) et l'occurrence de l'observation ( 1 si l'évènement a été observé et 0 , sinon).
Le BS est négativement orienté : plus sa valeur est petite, plus la performance du système évalué est bonne. Le score optimal de zéro est atteint pour une prévision déterministe (scénario unique) toujours correcte.

Le critère CSI est un score qui fait la synthèse entre le taux de fausses alertes et le taux de succès, déduits d'un tableau de contingence. Les tableaux de contingence indiquent le nombre de succès (évènements prévus et observés), d'évènements manqués (évènements non prévus mais observés), de fausses alertes (évènements prévus mais pas observés) et rejets corrects (évènements non prévus et non observés). Le CSI correspond au ratio entre le nombre de succès et la somme des succès, évènements manqués et fausses alertes. Le score CSI prend la valeur de 1 pour des « prévisions parfaites ». Ici, nous considérons un évènement prévu si $80 \%$ des scénarios de la prévision atteignent le seuil critique de débit étudié (quantile $90 \%$ ).

Tous les critères ont été calculés sur chaque bassin versant et sur l'ensemble des chroniques de débits observés et prévus disponibles. Les évaluations en contrôle et en prévision ont été faites sur la période avril 2005 à juillet 2009, période indépendante de la période de calage du modèle (1958 à 2000).

Dans ce qui suit, nous utilisons les performances du modèle GR3P (sans module neige) comme référence à laquelle comparer les résultats du modèle GR5P (avec module neige). Bien entendu, on s'attend à avoir des améliorations de performance entre ces deux modèles d'autant plus importantes que l'influence nivale est forte. Cependant, il sera important de vérifier la robustesse du modèle intégré qui a davantage de degrés de liberté, même lorsque la composante nivale est faible (correspondant alors à un faible niveau d'information pour estimer les paramètres du module neige)

\section{RESULTATS}

\section{III.1. Evaluation en contrôle (modèle forcé par les observations)}

Les résultats de l'évaluation en contrôle montrent une nette amélioration du critère de performance avec le modèle GR5P (GR3P avec module neige) (figure 2) : $64 \%$ des

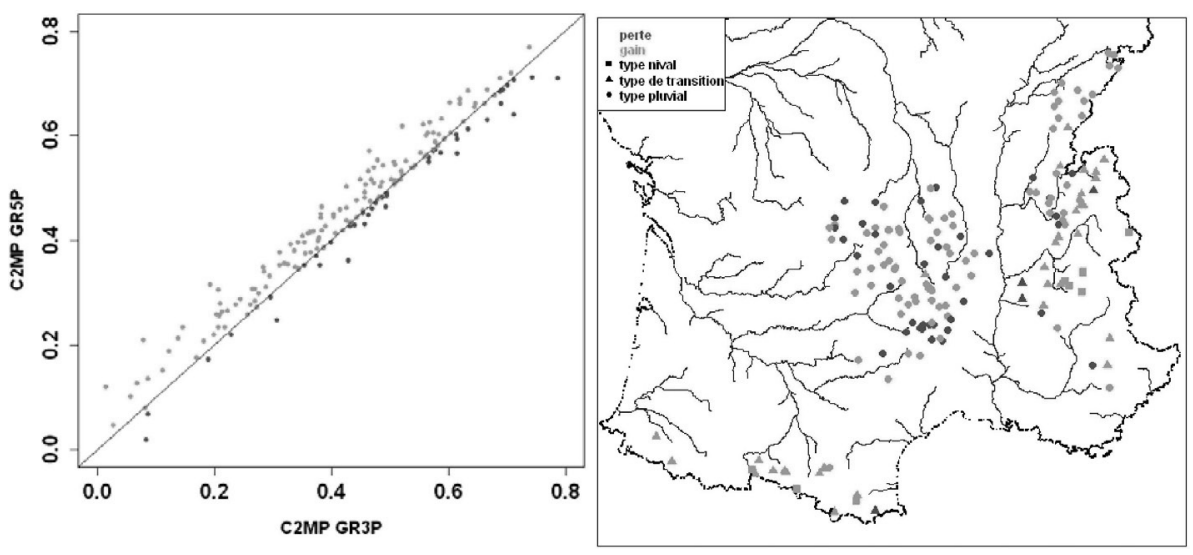

Figure 2 : Corrélogramme des performances de GR5P (modèle avec prise en compte de la neige) en fonction de GR3P (sans prise en compte de la neige) (à gauche) et carte des améliorations et dégradations des performances en fonction des régimes hydrologiques (à droite). Evaluation sur 176 bassins versants et pour la période d'avril 2005 à juillet 2009. Modèles forcés par les pluies observées. 

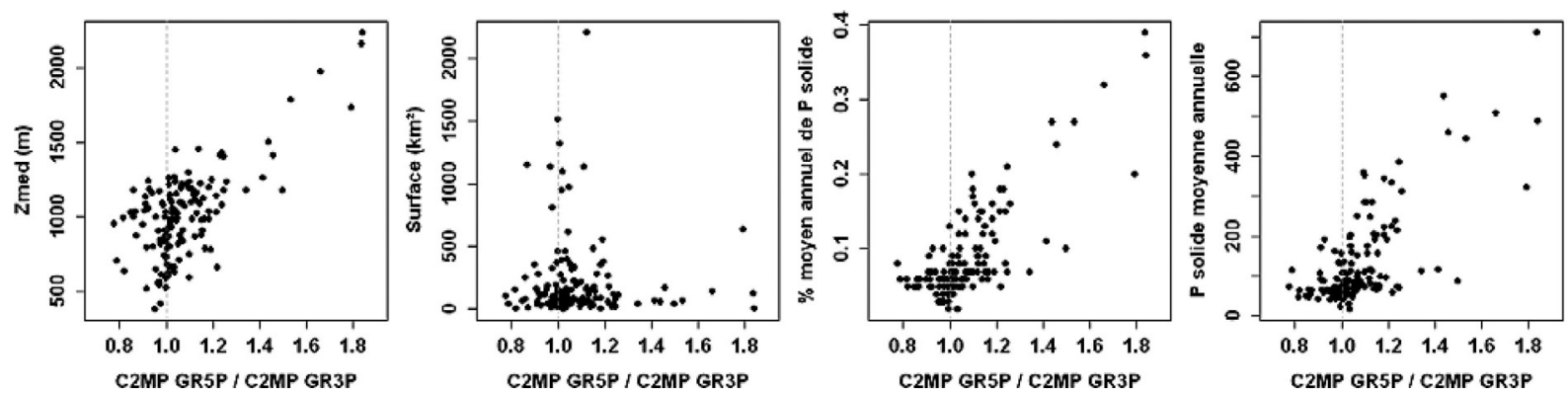

Figure 3 : Corrélogramme des variables physio-climatiques des bassins (de gauche à droite : altitude médiane, surface, pourcentage moyen annuel de précipitations solides, quantité moyenne annuelle de précipitation solide) en fonction du gain de performance. Modèles forcés par les pluies observées.

bassins (113 bassins) améliorent significativement leur critère et seulement $13 \%$ des bassins le dégradent (le restant de l'échantillon ne présentant pas une variation significative de la valeur du critère de performance). L'amélioration est observée pour tous les bassins à régime nival, tandis qu'elle touche $63 \%$ des bassins à régime pluvial et $92 \%$ des bassins caractérisés par un régime hydrologique de transition. Une analyse du gain apporté par le module neige en fonction des variables physio-climatiques des bassins (altitude médiane, surface, quantité annuelle moyenne de neige) (figure 3) a montré que les bassins où l'altitude médiane est inférieure à $600 \mathrm{~m}$ peuvent $\mathrm{s}$ 'affranchir du module neige pour une meilleure performance globale. La même tendance a été observée pour les bassins dont le pourcentage moyen annuel de précipitation solide (par rapport aux précipitations totales) est inférieur à $5 \%$. En revanche, les plus grands bassins de l'échantillon ont tendance à gagner en performance avec la prise en compte du module neige. Il en est de même pour ceux qui reçoivent, en moyenne, plus de $100 \mathrm{~mm} /$ an de précipitations sous forme neigeuse.

\section{III.2. Evaluation en prévision} (modèle forcé par les prévisions PEARP)

L'évaluation des prévisions hydrologiques d'ensemble montre également une amélioration des performances. La figure 4 montre le gain en performance évalué sur la base du score de probabilité ordonnée RPS. On observe l'apport positif du module neige pour près de $75 \%$ des bassins à régime nival et de transition pour l'échéance $\mathrm{j}+1$, et pour plus de $90 \%$ des bassins à régime nival pour l'échéance $\mathrm{j}+2$. Les bassins appartenant aux régimes de transition et nivaux sont ceux qui fournissent les plus importantes améliorations de performance, ce qui indique que le module remplit bien sa fonction.

La figure 5 illustre l'évaluation de la performance du système pour les dépassements du quantile $90 \%$ des débits journaliers, d'après les critères Brier Score (BS90) et CSI (CSI90). Les résultats sont également satisfaisants : l'intégration du module neige ne dégrade pas la performance du modèle GR3P. Au contraire, les performances du modèle intégré GR5P sont nettement meilleures, notamment pour les bassins à régime nival et de transition. L'apport de l'introduction du module neige dans la modélisation hydrologique est, en général, plus important pour l'échéance plus longue $(j+2)$, pour laquelle l'influence de la procédure de mise à jour du modèle hydrologique est moindre. Le critère CSI montre de très bonnes performances pour les bassins à régime dominant nival : à l'échéance $\mathrm{j}+2$, sa valeur moyenne est de 0,62 pour les bassins à régime nival, 0,46 pour les bassins de transition et 0,52 pour les bassins à régime pluvial.

Enfin, la figure 6 illustre les prévisions hydrologiques du modèle GR5P sur deux évènements particuliers. Nous
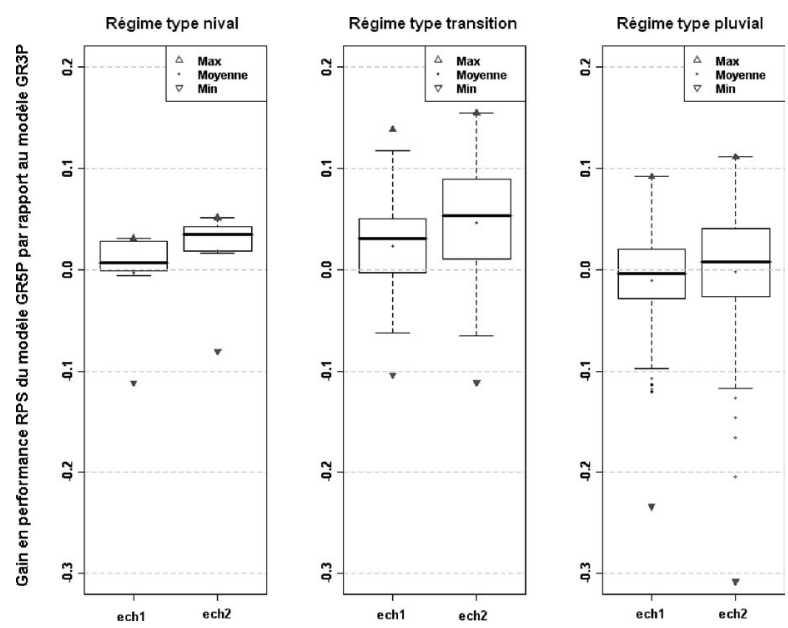

Figure 4 : Gain en performance du score RPS du modèle GR5P (avec module neige) par rapport au modèle GR3P (sans module neige) pour deux échéances journalières de prévision (ech1 $=j+1$; ech $2=j+2)$ et en fonction des régimes hydrologiques des bassins étudiés. Statistiques sur 176 basins versants et pour la période d'avril 2005 à juillet 2009. Boites à moustaches : le trait à l'intérieur représente la médiane des valeurs, les limites inférieures et supérieures des boîtes, les quantiles $25 \%$ et $75 \%$, et les limites inférieures et supérieures des moustaches, les quantiles $10 \%$ et $90 \%$. Modèles forcés par les prévisions d'ensemble PEARP de Météo-France. 

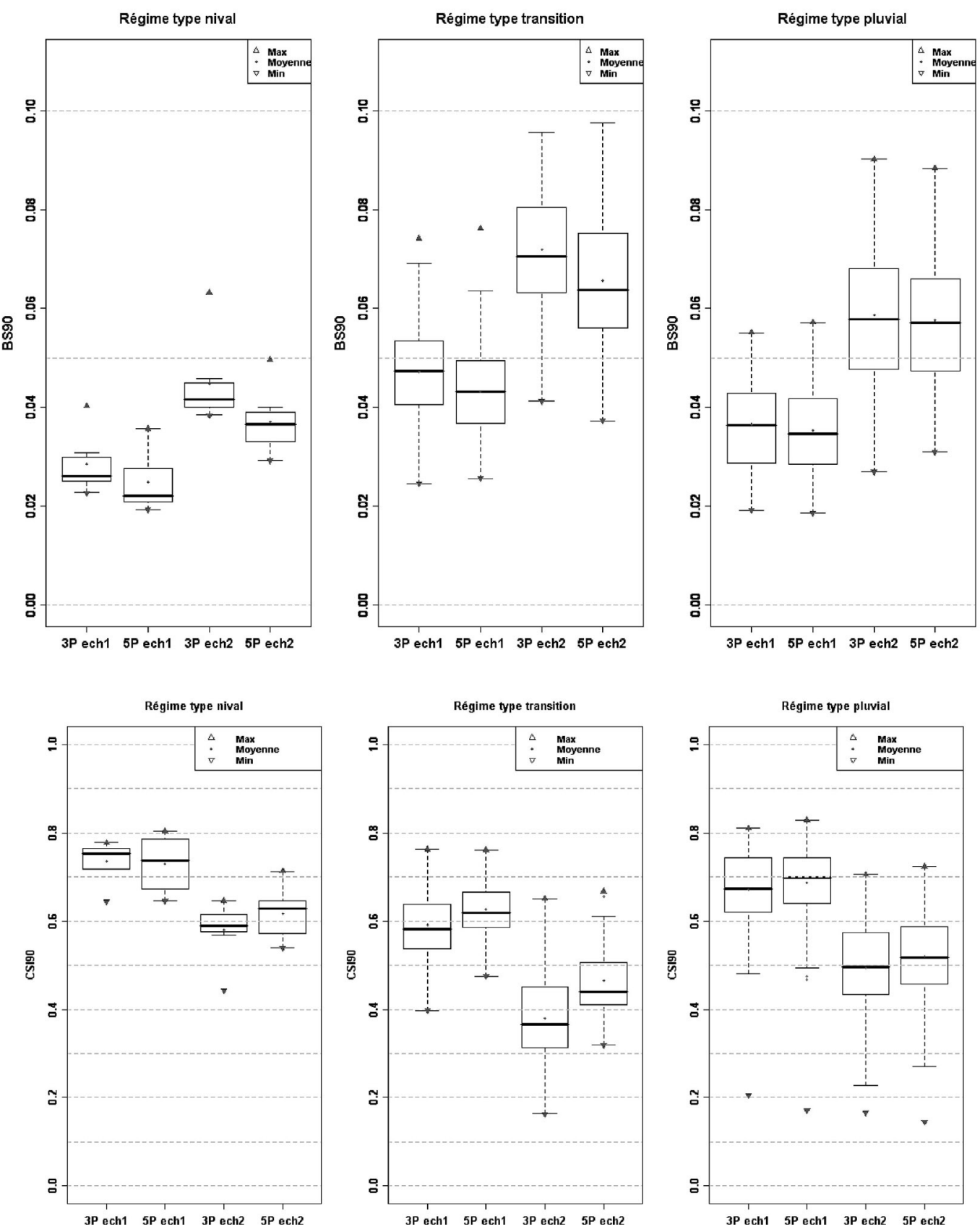

Figure 5 : Performances selon les critères Brier Score (en haut) et CSI (en bas) du modèle GR5P (avec module neige) et GR3P (sans module neige) pour deux échéances journalières de prévision (ech1 $=j+1$; ech $2=j+2$ ) et en fonction des régimes hydrologiques des bassins étudiés. Statistiques sur 176 basins versants et pour la période d'avril 2005 à juillet 2009. Boites à moustaches : idem figure 4. Modèles forcés par les prévisions d'ensemble PEARP de Météo-France

avons cherché à illustrer l'étude avec des évolutions de débits qui montrent une montée des eaux associée à peu ou pas de cumul de précipitations et à une augmentation des températures, afin de cibler des réponses hydrologiques liées très vraisemblablement à une fonte nivale uniquement. La figure 6-gauche illustre le cas du bassin de la Dore à Dorat $\left(1523 \mathrm{~km}^{2}\right)$ en mars 2005. La montée des eaux due à la fonte qui se déclenche aux alentours des 13-14 mars n'a pu être simulée que par le modèle GR5P avec prise en compte du stockage et de la fonte de la neige, même si le modèle surestime légèrement le débit de pointe. Le cas du bassin de la Séveraisse à Villar-Loubière $\left(133 \mathrm{~km}^{2}\right)$ (figure 6-droite), montre une décrue qui n'est pas assez soutenue au début du mois de mai 2005. Néanmoins, la montée des eaux déclenchée aux alentours du 24 mai est plutôt bien suivie par le modèle intégré. Enfin, on notera que, dans ce cas particuliers, les simulations obtenues avec le modèle forcé par les précipitations observées montrent aussi des écarts aux observations de débits, ce qui indique que des voies d'amélioration de la modélisation hydrologique restent ouvertes

\section{CONCLUSIONS}

Cette étude a permis d'évaluer les prévisions hydrologiques obtenues à partir d'une approche de modélisation qui intègre un module neige au sein d'un modèle de prévision hydrologique. Le module CemaNeige [Valéry, 2010] et le modèle de prévision hydrologique GR3P [Tangara, 2005] ont été utilisés. L'évaluation des prévisions du modèle intégré GR5P a été réalisée au pas de temps journalier et sur un échantillon de 176 bassins versants français situés dans des massifs 

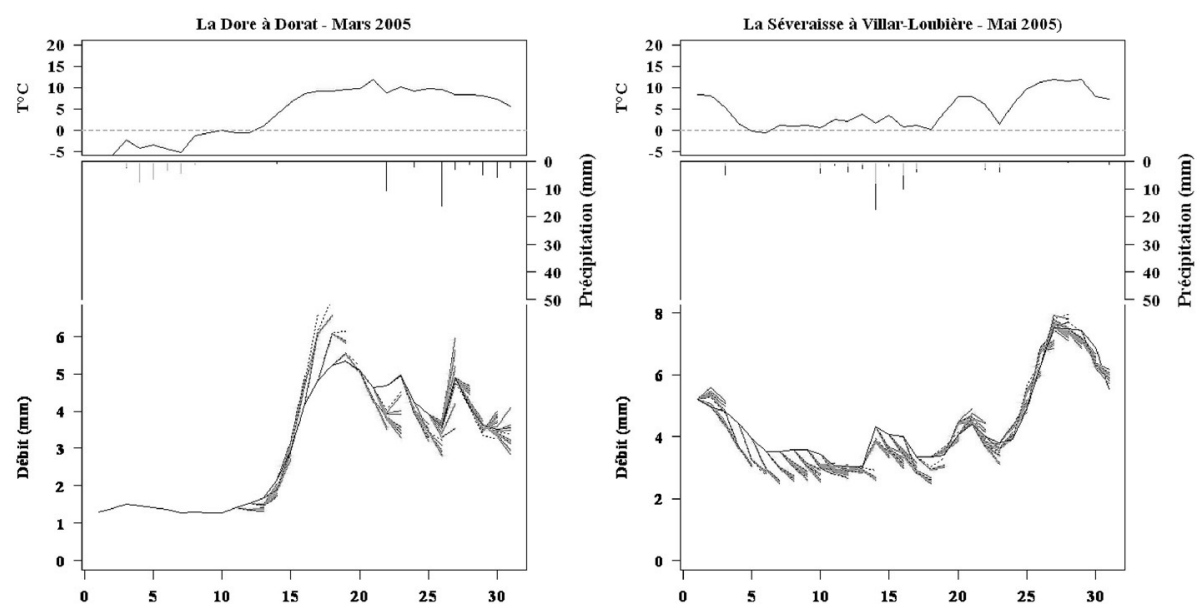

Figure 6 : Hydrogrammes des débits prévus par le modèle GR5P (avec prise en compte de la neige) quand le modèle est forcé par les précipitations observées (pointillés en noir) et par les 11 scénarios de la prévision d'ensemble (en vert), pour les bassins de la Dore à Dorat (1523 km²) en mars 2005 (à gauche) et de la Séveraisse à Villar-Loubière (133 km²) en mai 2005 (à droite). Les débits observés (en noir), les précipitations observées (pluie en bleue et neige en cyan) et les températures observées (graphique du haut, en rouge) sont également indiqués.

montagneux. La caractérisation des bassins versants en différents régimes hydrologiques nous a permis d'observer plus précisément quel pouvait être cet apport dans des conditions où la neige avait plus ou moins d'influence sur l'évolution des débits. Les performances du modèle ont été évaluées, d'une part, en considérant les précipitations observées comme scénario unique de précipitation future (modèle forcé par les observations), et, d'autre part, en utilisant les onze scénarios de prévision météorologique d'ensemble du système PEARP de Météo-France en entrée au modèle GR5P.

Les résultats de cette étude ont permis d'illustrer et quantifier, à partir d'un large échantillon de bassins versants, les gains de performance d'une modélisation intégrée hydronivale pour la prévision hydrologique comparativement à une modélisation hydrologique seule utilisée comme référence. La parcimonie du module neige (à deux paramètres) et du modèle pluie-débit (à trois paramètres) confère à l'ensemble une bonne robustesse, avec une amélioration globale des performances des prévisions hydrologiques lorsque l'évolution du stock et de la fonte de la neige est prise en compte. Ceci est vrai en particulier pour les bassins à régime à dominante nivale, ainsi que sur les plus grands bassins de l'échantillon et pour les bassins qui reçoivent, en moyenne, plus de $100 \mathrm{~mm} / \mathrm{an}$ de précipitations sous forme neigeuse. L'amélioration des débits prévus a été encore plus importante lorsque l'échéance de prévision augmentait (ici, pour l'échéance journalière $j+2$ ).

Cette étude a également abordé l'évaluation des performances des prévisions hydrologiques dans le cadre de la prévision d'ensemble, basée sur un ensemble de scénarios météorologiques futurs. Des scores probabilistes ont ainsi été utilisés pour évaluer la qualité des prévisions. Les résultats obtenus ont montré que l'intégration du module neige améliorait la qualité des prévisions et ne dégradait pas la performance du modèle GR3P. Ceci ouvre des perspectives pour l'intégration de cette modélisation intégrée dans des nombreux services opérationnels qui utilisent déjà le modèle pluiedébit GR3P pour leur prévision hydrologique. On soulignera ici que la synthèse et l'interprétation des résultats constituent une étape délicate dans la prévision d'ensemble. En effet, nombreux sont les critères qui permettent d'évaluer les performances d'un système de prévision. Les valeurs moyennes utilisées dans certains critères ne permettent pas de refléter la variabilité des résultats et la richesse d'information donnée par les différents scénarios de la prévision d'ensemble. Les performances des modèles sont ainsi assez complexes à analyser, du fait de la complexité même des critères et du caractère probabiliste des prévisions.

En conclusion, l'introduction d'un module neige au sein du modèle pluie-débit GR3P apporte des améliorations conséquentes pour la prévision hydrologique. Même quand les paramètres libres du module neige sont fixés à des valeurs moyennes obtenues sur la France [Valéry, 2010], les performances restent très satisfaisantes (voir Nicolle [2010]). Ceci ouvre des perspectives intéressantes pour des études et applications opérationnelles sur des bassins versants non jaugés, où l'estimation des paramètres est une tâche délicate. De plus, des pistes de recherche restent à explorer ou à approfondir. Il serait intéressant, par exemple, de tester d'autres critères d'évaluation qui cibleraient plus particulièrement la qualité des prévisions des épisodes de crue, ou encore d'examiner un grand nombre d'évènements critiques pour pouvoir mieux quantifier la part des différentes sources d'incertitudes dans la prévision hydrologique.

\section{REFERENCES ET CITATIONS}

Bergström S. (1975) - Development of a snow routine for the HBV-2 model. Nordic Hydrology. 6(2) 73-92

Dhi (2009) - MIKE 11. A Modelling System for Rivers and Channels, Reference Manual

Garçon R. (1996) - Prévision opérationnelle des apports de la Durance à Serre-Ponçon à l'aide du modèle MORDOR. Bilan de l'année 1994-1995. La Houille Blanche. 5 71-76

Garçon R. (1999) - Modèle global pluie-débit pour la prévision et la prédétermination des crues. La Houille Blanche. 7/8 88-95

Garen D.C., Marks D. (2005) - Spatially distributed energy balance snowmelt modelling in a mountainous river basin : estimation of meteorological inputs and verification of model results. Journal of Hydrology. 315(1-4) 126-153

Gottardi F. (2009) - Estimation statistique et réanalyse des précipitations en montagne -Utilisation d'ébauches par types de temps et assimilation de données d'enneigement- application aux grands massifs montagneux français. Thèse de Doctorat, INPG (Grenoble), France. $261 \mathrm{pp}$ 
Klemeŝ V. (1990) — The modelling of mountain hydrology : The ultimate challenge. IAHS-AISH Publication. 190 29-43

Lehning M., Völksch I, Gustavson D, Nguyen Ta, Stähli M, Zappa M. (2006) - ALPINE3D : a detailed model of mountain surface processes and its application to snow hydrology. Hydrological processes. 20 2111-2128

Lindström G., Johansson B., Persson M., Gardelin M., Bergström S. (1997) - Development and test of the distributed HBV-96 hydrological model. Journal of Hydrology. 201(1-4) 272-288

Mathevet T., Michel C., Andréassian V., Perrin C. (2006) - A bounded version of the Nash-Sutcliffe criterion for better model assessment on large sets of basins. IAHS-AISH Publication. 307 211-219

Morin G. (2002) - CEQUEAU hydrological model. In : V.P. Singh, D.K. Frevert (Editors), Mathematical models of large watershed hydrology. Water Resources Publications, Highlands Ranch, Colorado. 507-576

Nicolau J. (2002) - Short-range ensemble forecasting. In Proc. WMO/CBS Technical Conferences On Data Processing and Forecasting Systems, Cairns, Australia, 2-3 Dec. 4 p

Nicolle P. (2010) — Prise en compte de la neige dans la prévision hydrologique : quel apport pour mieux prévoir les crues nivales. Mémoire de Master, Université Pierre et Marie Curie, Cemagref, Paris. $53 \mathrm{pp}$

Oudin L., Hervieu F., Michel C., Perrin C., Andreassian V., Anctil F., Loumagne C. (2005) - Which potential evapotranspiration input for a rainfall-runoff model ? Part 2 - Towards a simple and efficient PE model for rainfall-runoff modelling. Journal of Hydrology. 303 (1-4) 290-306

Paquet E. (2004) — Evolution du modèle hydrologique MORDOR : modélisation du stock nival à différentes altitudes. La Houille Blanche. 2 75-82

Quintana-Segui P., Le Moigne P., Durand Y., Martin E., Habets F., Baillon M., Canellas C., Franchisteguy L., Morel S. (2008) Analysis of near-surface atmospheric variables : Validation of the
SAFRAN analysis over France. Journal of Applied Meteorology and Climatology. 47 (1) 92-107

Ramos M.H., Thielen J., Pappenberger F. (2008) - Utilisation de la prévision météorologique d'ensemble pour la prévision opérationnelle et l'alerte aux crues. Proc. Colloque SHF Prévisions hydrométéorologiques, Lyon, 18-19/11/2008. 191-199

Randrianasolo A., Ramos M.H., Thirel G., Andreassian V., Martin E. (2010) - Comparing the scores of hydrological ensemble forecasts issued by two different hydrological models. Atmospheric Science Letters. 11 100-107

Sauquet E. (2006) - Cartographie des écoulements moyens mensuels en France. Note de synthèse Cemagref. 92 pp

Tangara M. (2005) - Nouvelle méthode de prévision de crue utilisant un modèle pluie-débit global. Thèse de doctorat, EPHE, Cemagref, Paris. 374 pp

Thirel G., Rousset-Regimbeau F., Martin E., Habets F. (2008) On the impact of short-range meteorological forecasts for ensemble streamflow prediction. Journal of Hydrometeorology. 9 1301-1317

Turcotte R., Fortin L.G., Fortin V., Fortin J.P., Villeneuve J.P. (2007) - Operational analysis of the spatial distribution and the temporal evolution of the snowpack water equivalent in southern Québec, Canada. Nordic Hydrology. 38(3) 211-234

Valery A. (2010) - Modélisation précipitations-débit sous influence nivale, élaboration d'un module neige et évaluation sur 380 bassins versants.Thèse de doctorat, ENGREF, Cemagref, Paris. 405 pp

Vidal J.-P., Martin É., Franchisteguy L., Baillon M., Soubeyroux J.-M. (2009) - A 50-year high-resolution atmospheric reanalysis over France with the Safran system. International Journal of Climatology. 30 1627-1644

Wilks D. S. (2011) - Statistical Methods in the Atmospheric Sciences. $3^{\text {rd }}$ Edition, Academic Press. 676 pp

Zappa M., Pos F, Strasser U, Warmerdam P, Gurtz J. (2003) Seasonal Water Balance of an Alpine Catchment as Evaluated by Different Methods fo Spatially Distributed Snowmelt Modelling. Nordic Hydrology. 34(3) 179-202 